\title{
REPRESENTACIÓN DE CIUDADANÍA Y LA CONFIANZA EN LA DEMOCRACIA SEGÚN EL NIVEL EDUCATIVO EN JÓVENES DE 18 A 23 AÑOS EN LIMA METROPOLITANA
}

\author{
REPRESENTATION OF CITIZENSHIP AND THE CONFIDENCE IN THE \\ DEMOCRACY ACCORDING TO THE EDUCATIVE LEVEL IN YOUNG PEOPLE OF \\ 18 TO 23 YEARS IN LIMA METROPOLITAN
}

\author{
CARLOS ALBERTO ARENAS IPARRAGUIRRE ${ }^{\mathbf{1}}$, HÉCTOR HERNÁNDEZ VALZ, \\ María L. MATALINARES CALVET, JOSÉ CARLOS RIVERA BENAVIDES \\ Universidad Nacional Mayor de San Marcos, Facultad de Psicología
}

\section{RESUMEN}

OBJETIVO: Conocer la representación de la ciudadanía y la confianza de la democracia según el nivel educativo en jóvenes de 18 a 23 años de Lima Metropolitana. DISEÑO: Comparativo transversal. La población estuvo conformada por jóvenes entre 18 a 24 años estudiantes de los últimos grados de instrucción secundaria y últimos ciclos académicos de dos universidades nacionales, con un muestreo de tipo intencional no probabilística. MATERIAL Y MÉTODOS: Los instrumentos utilizados fueron la técnica del Focus Groups para la representación de la ciudadanía y el Rating 5cale para la confianza en las instituciones democráticas. RESULTADOS: El análisis de los resultados fue mediante cálculos porcentuales respecto a los indicadores de la representación ciudadana: encontrando diferencia entre los estudiantes de secundaria y los universitarios, en los primeros el DNI es la representación de ciudadanía, en los universitarios está enmarcado dentro de los Derechos y Deberes; sin embargo, cerca de un 10\% no presentan un nivel de abstracción diferentes a los del nivel secundario. En cuanto a la confianza en la democracia se aprecia que no hay diferencia significativa entre ambos grupos de estudiantes. CONCLUSIONES: Se concluyó que el nivel de confianza en la democracia de los jóvenes escolares y de estudiantes universitarios es bajo, expresado en identificar al gobierno actual con el sistema democrático, la representación de ciudadanía de los jóvenes escolares es diferente a los estudiantes universitarios, debido al pronto ingreso, al campo laboral y al compromiso personal que tienen, teniendo el nivel educativo un efecto directo sobre la confianza en la democracia: el estudiante universitario tiene una mayor preocupación por los problemas del país y por el papel que le corresponde a cada individuo en la esfera de las decisiones públicas que el joven escolar quienes no problematizan el ejercicio de sus derechos y muy pocos expresan nociones relacionadas con la confianza en la democracia.

Palabras claves: Democracia, Ciudadanía, Representaciones Sociales, Confianza.

\begin{abstract}
Objective: To know the representation the citizenship and the confidence of the democracy according to the educative level in young people of 1823 years of Lima Metropolitan. DESIGN: Comparative cross-sectional. The population was confirmed by young people between 18 to 24 years students of the last secondary level of trainings and last academic cycles of two national universities, with a no probabilistic sampling of intentional type. MATERIAL And METHODS: The used instruments were the technique of the Focus Groups for the representation of the citizenship and the Scale Rating for the confidence in

${ }^{1}$ Email: carenasi@unmsm.edu.pe
\end{abstract}


the democratic institutions. RESULTS: The analysis of the results was by means of percentage calculations with respect to the indicators of the citizen representation: finding difference between the students of secondary and the college students, in the first DNI it is the representation of citizenship, in the college students it is framed within the Rights and Duties; nevertheless, near a $1 \%$ they do not present display a level of abstraction different from those from the secondary level. As far as the confidence in the democracy it is appraised that there is no significant difference between both groups of students. CONCLUSIONS: One concluded that the level of confidence in the democracy of the scholastic young people and of university students is low, expressed in identifying to the present government with the democratic system, the representation of citizenship 01 the scholastic young people is different from the university students, due to soon the entrance to the labor field and the personal commitment that they have, having the educative level a direct influence on the confidence in the democracy: the university student has a greater preoccupation by the problems of the country and by the paper that corresponds to him to each individual in the sphere of the public decisions that the young student who do not problematical the exercise of their rights and very few express slight knowledge related to the confidence in the democracy.

Keywords: Democracy, Citizenship, Social Representation, Confidence

\section{INTRODUCCIÓN}

La relación entre la representación de la ciudadanía y la confianza en la democracia es un tema que, en los últimos años, se ha tornado vigente y de preocupación de múltiples sectores de la sociedad, incluyendo las diversas disciplinas, científico-sociales, los políticos y para la propia sociedad civil en su conjunto. La presión de estas afirmaciones se confirma con muchos estudios realizados en América Latina, en los que se constata la preferencia de las mayorías sociales por los regímenes democráticos. Pero la participación política no es sólo el canal de expresión de la ciudadanía, también se presentan en espacios propios de la sociedad civil, de nivel regional y local.

Son los jóvenes de ahora los que van a ser los protagonistas de la futura sociedad peruana y latinoamericana y por ello puede ser interesante conocer cuáles son las presentaciones sociales que ellos tienen acerca de la Ciudadanía y su confianza en La Democracia. A partir de estos presupuestos el propósito del estudio fue conocer la representación de la ciudadanía del nivel educativo sobre la confianza en democracia en jóvenes de 18 a 23 años los últimos años de estudios secundarios de los últimos años de los estudios universitarios en diversos centros de estudios. Se pidió a dichos estudiantes que respondieran a dos instrumentos: un cuestionario que combinaba el enfoque de Lopez, S. ${ }^{1}$ sobre Mapas de Ciudadanía en el Perú por medio de la técnica de Grupos focales en relación a las Actitudes hacia la ciudadanía elaborándose un cuestionario de Representación de Ciudadanía y la Escala de Representación de Democracia: Rating Scale para la confianza en las instituciones democráticas. Desde el enfoque sobre las Representaciones Sociales de Moscovici S. ${ }^{2}$

Estudios previos en Perú sobre las actitudes hacia la democracia en estudiantes universitarios realizado por Ponce, C. y Aliaga, J. ${ }^{3}$ en su estudio realizado en la Universidad Nacional Mayor de San Marcos, evidencian la fragilidad de la democracia de la que emana el imperativo de formar ciudadanos con personalidad democrática que 
mediante la participación la protejan de todo riesgo y la fortalezcan. Esto requiere de una cultura de la democracia, de una cultura cívica, íntimamente vinculada a la cultura de la participación, a un actitud política viva que se desarrolla y consolida cuando los ciudadanos asumen como parte de su vida política los valores y las instituciones democráticas. También mencionan otras evidencias en la que jóvenes de diversos sectores de Lima no tienen una mayor identificación con la democracia y que poseen elevados niveles de escepticismo además de la creencia que la democracia o su ausencia no cambia su situación y puede estar relacionada con la connotación negativa que la política y los políticos tienen para los estudiantes evaluados. Otra aproximación peruana al tema es la realizada por Murakami, Y. ${ }^{4}$, donde realiza un estudio sobre el poder visto desde abajo, centrada en las opiniones de los sectores $\mathrm{C}$ y D evidenciando que muchas de las personas de estos sectores socioeconómico delegan en los dirigentes la parte importante de toma de decisiones y subsiste la tendencia «plebiscitaria» en la conciencia política popular, existe en ellos también una baja lealtad y apego a la democracia que en otros sectores, confirmación que coincide con un enfoque escéptico de la democracia en los sectores populares, de este modo «los sectores populares captan el sentido de la participación política desde la perspectiva utilitaria y no han asimilado la participación democrática como un valor propio».

La representación social es un sistema de valores, de nociones y de prácticas relativas a objetos, aspectos o dimensiones del medio social, que permite, no solamente la estabilización del marco de vida de los individuos y los grupos, sino que constituye también un instrumento de orientación de la percepción de situaciones y de la elaboración de respuestas. La investigación en representaciones sociales a menudo se focaliza en contenidos específicos de sistemas de conocimiento que caracterizan grupos sociales y sociedades Las representaciones sociales tienen tres componentes básicos: Información, Campo de representación, Actitud.

Más allá de la ciudadanía otorgada creemos que existe una ciudadanía conquistada. Sustentamos la presente investigación en la tesis de la ciudadanía como desarrollo de la igualdad de condiciones y como base de la democracia moderna, de igual modo definimos al ciudadano como un individuo con derechos garantizados por el Estado y con responsabilidades hacia la comunidad política de la que forma parte.(dimensión activa y pasiva).

Para López, $\mathrm{S}^{5}$. No hay un concepto único de ciudadanía. En realidad hay tantos tipos de ciudadanía como tipos de comunidades políticas modernas. Existe el ciudadano liberal en sus diversas variantes, el ciudadano social demócrata, el fascista ,el comunista ,etc. Estas diferencias se sustentan en los principales análisis y debates teóricos que sobre la ciudadanía se ha producido en tres diferentes coyunturas intelectuales y políticas en la historia del mundo moderno. El primer debate que 'se produjo en los albores de la era moderna tenía como objetivo desentrañar el sentido y las características del hombre en su relación con la sociedad y con el Estado Moderno. Dando lugar a concepciones liberales y socialistas de la ciudadanía. Segundo debate los estudios contemporáneos sobre la problemática de la ciudadanía se intensificaron, estas concepciones fueron inaugurados por T.H. Marshall, quien al analizar en 1949 esta categoría como conquista de derechos y como espacio de conflicto entre dos principios contrapuestos (el de la igualdad de la democracia y el de la desigualdad de la economía capitalista ciudadanía social como punto culminante del desarrollo histórico de la ciudadanía moderna) sentó las bases de una concepción socialdemócrata de la ciudadanía. Norberto Bobbio y Albert O. Hirschman contribuyen a desarrollar los puntos de vista de Marshall, han aportado en el campo de la concepciones de ciudadanía en la naturaleza del capital ismo contemporáneo además y principalmente en el de las condiciones, actores y las estrategias de construcción ciudadana. La extensión de la ciudadanía a las clases bajas implicó una institucionalización de criterios abstractos de 
igualdad que dieron origen a nuevas desigualdades. Estas fueron mitigadas por renovadas soluciones de compromiso siempre parciales. El proceso estuvo marcado por la permanente tensión entre la idea plebiscitaria y la idea de la representación global. El tercer debate, en los setenta y ochenta sobre los temas fundacionales de la sociedad moderna: la naturaleza del individuo y de sus derechos en su relación con el Estado, la comunidad y la cultura, la cuestión de la ética y los fundamentos de la filosofía política. Este nuevo debate enfrentó a diversos protagonistas cuyo núcleo polémico: los liberales encabezados por Rawls, y los comunitaristas por Hannah, Arendt, Michael Walzer, Charles Taylor.

Existen diversas concepciones de ciudadanía, ello supone una serie de elementos básicos que la definen: El ciudadano es un individuo que no tiene relaciones de dependencia personal o que ha roto con ellas y que por eso mismo es relativamente autónomo. El ciudadano es un individuo con derechos que son enfatizados frente a las tradicionales responsabilidades que imponían los estados pre-modernos. Ello implicó diferentes formas de definir los derechos: (a) La capacidad de actuar libremente sin impedimentos que bloquean la acción ni restricciones que obligan a actuar de una manera no deseada, (b) la capacidad de participar en la riqueza y el bienestar que produce una sociedad ha dado lugar a los derechos sociales, (c) el ciudadano con derechos implica una peculiar relación de la sociedad y de los individuos con el Estado moderno que reconoce un claro predominio de los derechos sobre las responsabilidades y ofrece una serie de garantías constitucionales organizativas e institucionales para concretarlos, (e) la ciudadanía implica un sentido de pertenencia y de membrecía a una determinada comunidad política entre cuyos miembros se establecen relaciones de interdependencia, responsabilidad, solidaridad y lealtad, habría que incluir uno nuevo (d) las aportaciones de la Sociedad de la Información y el Conocimiento en cuanto a la accesibilidad de la información, juntamente con una sociedades cada vez más instruidas, nos aportan una ciudadanía más eficiente. Por tanto, el acceso a la información se convierte en un factor clave para asegurar una participación ciudadana reflexiva e informada.

Algunos autores latinoamericanos concluyen al respecto que la distribución social del conocimiento está atravesada por una profunda desigualdad, y la brecha entre el experto, el ciudadano bien informado y el hombre común, no solo no se ha reducido sino que se ha profundizado.

Para Franco, C. ${ }^{6}$ el problema es que para que el principio de la igualdad política, propugnada por la ciudadanía, funcione es preciso que se reduzca de tal modo la desigualdad real entre los hombres que haga posible que estos sigan creyendo que vale la pena luchar por la igualdad

Los mapas de la ciudadanía, formulados por López, $\mathrm{S}^{5}$ se analizan tres aspectos relacionados con ella: los niveles de la ciudadanía, la consistencia ciudadana y las brechas ciudadanas asociados con la modernización, la democratización y la pobreza constituyéndose los índices de ciudadanía. En primer lugar los peruanos no tenemos el mismo nivel de ciudadanía. En segundo lugar no todos los peruanos tenemos igual acceso a todos los derechos ciudadanos, más de la mitad de los peruanos (53\%) y el 90,2\% de los distritos tienen bajos y muy bajos niveles de consistencia ciudadana En tercer lugar, las brecha ciudadanas - género, regionales, rural urbanas, étnicas- son mayores en los departamentos que tienen bajos niveles de modernización y e democratización y mayores niveles de pobreza En cuarto lugar, son los distritos de la sierra, especialmente del sur andino, los que presentan una aguda situación de déficit de ciudadanía. Ellos tienen los más bajos niveles de ciudadanía, las más bajas consistencias ciudadanas y las más altas brechas ciudadanas.

El régimen democrático es la forma de vida política que da la mayor libertad al mayor 
número, que protege y reconoce la mayor diversidad posible La Democracia en América Latina ha sido estudiada en profundidad, a la luz de los últimos resultados del Eurobarómetro y el Latinobarómetro se puede ver que el apoyo a la democracia por parte de la población latinoamericana ha bajado preocupantemente en los últimos años (2001). A la pregunta sobre qué tipo de régimen es preferible, sólo un 48 por ciento de los encuestados respondieron que era la Democracia.

Parece difícil entender la democracia en el mundo moderno sin remitirse a la representación. Los canales nunca deben ser obligatorios, cada uno debe poder elegir cuál es su implicación en el proceso político. Tal y como sostiene algunos autores, el exceso de politización puede desembocar en el efecto contrario, la reivindicación de lo privado, lo que se debe realizar es la generación de nuevos espacios de discusión y toma de decisión. Tal y como sostiene Bobbi0 ${ }^{7}$ se trata de democratizar la sociedad. Esto es, que la mayor parte de las instituciones de la sociedad tengan canales democráticos de participación y deliberación, a la par que haya una apertura de las instituciones gubernamentales para que sean permeables a las propuestas de los foros de discusión elaborados para tal fin.

Estas condiciones necesarias tendrán sentido, desde nuestro punto de vista si se presenta simultáneamente la confianza, la lealtad, el apego y la conciencia de ello por la ciudadanía para convertir las categorías y procesos de democracia y ciudadanía en constantes de la vida personal y social.

Es desde la perspectiva y enfoque asumidos que se planteó la necesidad de explicar el ien función al nivel educativo, ¿difiere la representación de ciudadanía y la confianza en la democracia en jóvenes estudiantes de 18 a 23 años de Lima-Metropolitana?, los objetivos necesarios fueron: conocer el nivel de confianza en la democracia de los jóvenes estudiantes de acuerdo a nivel educativo, el describir la representación de ciudadanía de los jóvenes estudiantes de LimaMetropolitana, de acuerdo a su nivel educativo y culminar evidenciando la influencia de la representación de la ciudadanía y el nivel educativo sobre la confianza en la democracia.

\section{Variables}

- Independiente:

Nivel Educativo

- Dependiente:

Representación de ciudadanía Confianza en la democracia

- Controladas:

Estudiantes 5to Secundaria y estudiantes universitarios

\section{Hipótesis}

- La representación de la ciudadanía difiere según el nivel educativo en estudiantes de Lima Metropolitana

- La confianza en la democracia difiere según el nivel educativo en estudiantes de Lima Metropolitana

\section{MATERIALES Y MÉTODOS}

Tipo de investigación aplicada, con la metodología descriptiva y diseño comparativo transversal.

La población estuvo conformada por los jóvenes entre 18 a 24 años estudiantes de los últimos años de secundaria y primeros ciclos de diversas universidades . 
Muestra

Se efectuó un muestreo de tipo intencional no probabilística.

Criterios de selección de la muestra

- Estratificación de dos niveles educativos.

- Estudiantes con secundaria completa

- Estudiantes de los últimos ciclos universitarios

\section{Instrumentos}

Se utilizaron dos instrumentos de recolección de datos; el primero para obtener información acerca de la representación de ciudadanía mediante la técnica del Focus Group, para lo cual se elaboró un cuestionario con respecto a la Representación de Ciudadanía basado en el modelo expuesto por López ${ }^{5}$ sobre la existencia de un Mapa de Ciudadanía entre la población peruana. Dicho cuestionario estuvo formado por las siguientes preguntas.

1) ¿En qué consiste ser ciudadano en el Perú?

2) ¿Te sientes ciudadano en el Perú?

3) ¿Qué tareas te esperan como ciudadano del Perú?

Se hizo una prueba piloto para determinar si en especial los alumnos de secundaria comprendían las preguntas, con 20 alumnos y confirmaron que las preguntas eran claras. El resultado final del focus group es de tipo categorial y consiste en las siguientes:

1) Reconoce que tiene derechos o no lo hace.

2) Es un ciudadano completo o no lo es.

3) Afrontar los problemas nacionales o vivir su vida personal ya.

El segundo instrumento fue para medir la confianza en la democracia para lo cual se elaboró una escala ad hoc: Rating Scale para la confianza en las instituciones democráticas El rating scale es un instrumento que permite al sujeto asignar una calificación sobre un número de grados elegido de acuerdo con el objetivo.

Para esta investigación se eligió una escala del 1 al 10 y los reactivos estuvieron constituidos por las instituciones del gobierno y la confianza que inspiran en la población. Arroja resultados cuantitativos que son tratados con estadísticas paramétricas.

\section{RESULTADOS}

El conocimiento de la representación ciudadana utilizando el focus group, teniendo como elementos de análisis, meditación, críticas, opiniones las siguientes preguntas motivadoras: Pregunta 1 ¿En qué consiste ser ciudadano en el Perú?

Pregunta 2 ¿Te sientes ciudadano en el Perú? Pregunta 3 ¿Qué tareas te esperan como ciudadano del Perú?

Luego se anotaron las síntesis de cada sesión las que se dieron lectura con el propósito de confirmar la interpretación hecha por escrito, sólo si los participantes estaban de conformidad con el texto leído se aceptaba como respuesta concluida. Los resultados pueden verse en la tabla 1, tanto para el grupo de estudios secundarios como para los universitarios, en la misma tabla presentamos los resultados de la confianza en la 
democracia, estos proceden del rating scale, que tiene como indicadores las instituciones democráticas (el congreso, el ejecutivo, fiscalía, presidente de la república, sistema electoral, fuerzas armadas, defensoría del pueblo, tribunal constitucional) y de la sociedad civil (sindicatos, municipalidades). Los resultados se presentan debajo del rotulo pregunta 1 (preg1): R1 Tener el DNI; pregunta 2 (preg2); R2 Tener derechos; pregunta 3 (preg3): R3 Tener derechos y obligaciones, y para el rating scale, se presenta el puntaje obtenido por cada examinado. Al pie de la tabla presentamos las frecuencias registradas según el tipo de respuesta para cada una de las preguntas. 
TABLA 1: Respuestas encontradas al focus group y la escala de confianza en la democracia en la muestra de estudiantes de secundaria y universitarios

\begin{tabular}{cc}
\multicolumn{2}{c}{ SECUNDARIA } \\
\hline $\begin{array}{c}\text { Representación } \\
\text { Ciudadana }\end{array}$ & $\begin{array}{c}\text { Confianza en } \\
\text { la } \\
\text { Democracia }\end{array}$
\end{tabular}

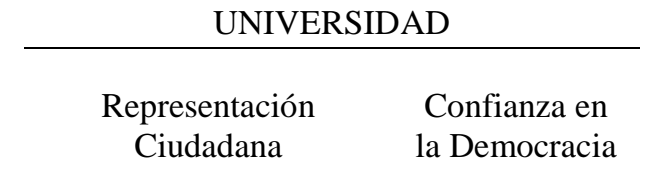

\begin{tabular}{|c|c|c|c|c|c|c|c|c|c|}
\hline sujetos & Preg.1 & Preg. 2 & Preg. 3 & Ptje. & sujetos & Preg.1 & Preg. 2 & Preg.3 & Ptje. \\
\hline 1 & 1 & 1 & 2 & 40 & 1 & 2 & 2 & 2 & 54 \\
\hline 2 & 1 & 1 & 1 & 38 & 2 & 2 & 2 & 2 & 37 \\
\hline 3 & 1 & 1 & 1 & 45 & 3 & 2 & 2 & 2 & 29 \\
\hline 4 & 1 & 1 & 1 & 58 & 4 & 2 & 2 & 2 & 35 \\
\hline 5 & 1 & 1 & 1 & 39 & 5 & 2 & 2 & 1 & 38 \\
\hline 6 & 1 & 1 & 1 & 46 & 6 & 3 & 2 & 1 & 41 \\
\hline 7 & 1 & 1 & 2 & 30 & 7 & 2 & 2 & 1 & 46 \\
\hline 8 & 1 & 1 & 1 & 62 & 8 & 2 & 2 & 1 & 35 \\
\hline 9 & 1 & 1 & 1 & 37 & 9 & 1 & 2 & 1 & 54 \\
\hline 10 & 1 & 1 & 1 & 48 & 10 & 2 & 2 & 1 & 42 \\
\hline 11 & 1 & 1 & 1 & 50 & 11 & 2 & 1 & 1 & 47 \\
\hline 12 & 1 & 1 & 1 & 37 & 12 & 2 & 1 & 1 & 34 \\
\hline 13 & 1 & 1 & 1 & 42 & 13 & 2 & 1 & 1 & 43 \\
\hline 14 & 1 & 1 & 1 & 47 & 14 & 3 & 1 & 2 & 45 \\
\hline 15 & 1 & 1 & 1 & 34 & 15 & 2 & 1 & 2 & 49 \\
\hline 16 & 1 & 1 & 1 & 43 & 16 & 2 & 1 & 2 & 35 \\
\hline 17 & 1 & 1 & 2 & 45 & 17 & 2 & 1 & 2 & 45 \\
\hline 18 & 1 & 1 & 1 & 54 & 18 & 3 & 1 & 2 & 24 \\
\hline 19 & 1 & 1 & 1 & 35 & 19 & 2 & 1 & 2 & 48 \\
\hline 20 & 1 & 1 & 1 & 45 & 20 & 2 & 1 & 1 & 60 \\
\hline 21 & 1 & 1 & 1 & 36 & 21 & 2 & 1 & 1 & 54 \\
\hline 22 & 1 & 1 & 1 & 49 & 22 & 3 & 1 & 1 & 44 \\
\hline 23 & 1 & 1 & 1 & 25 & 23 & 1 & 1 & 2 & 54 \\
\hline 24 & 1 & 1 & 1 & 36 & 24 & 2 & 1 & 2 & 42 \\
\hline 25 & 1 & 1 & 1 & 24 & 25 & 2 & 2 & 2 & 42 \\
\hline 26 & 1 & 1 & 2 & 48 & 26 & 2 & 2 & 2 & 57 \\
\hline 27 & 1 & 1 & 1 & 60 & 27 & 3 & 2 & 1 & 45 \\
\hline 28 & 1 & 1 & 1 & 54 & 28 & 3 & 2 & 1 & 34 \\
\hline 29 & 1 & 1 & 1 & 44 & 29 & 2 & 2 & 1 & 56 \\
\hline 30 & 1 & 1 & 1 & 54 & 30 & 2 & 2 & 1 & 45 \\
\hline 31 & 1 & 1 & 1 & 37 & 31 & 1 & 1 & 1 & 46 \\
\hline 32 & 1 & 1 & 1 & 42 & 32 & 2 & 1 & 2 & 42 \\
\hline 33 & 1 & 1 & 2 & 57 & 33 & 3 & 1 & 2 & 41 \\
\hline 34 & 1 & 1 & 1 & 45 & 34 & 2 & 1 & 2 & 42 \\
\hline 35 & 1 & 1 & 1 & 34 & 35 & 2 & 1 & 1 & 42 \\
\hline 36 & 1 & 1 & 1 & 66 & 36 & 3 & 1 & 2 & 42 \\
\hline Frec de 1 & 36 & 36 & 31 & & Frec de 1 & 3 & 20 & 18 & \\
\hline Frec de 2 & 0 & 0 & 5 & & Frec de 2 & 25 & 16 & 18 & \\
\hline Frec de 3 & 0 & 0 & 0 & & Frec de 3 & 8 & 0 & 0 & \\
\hline
\end{tabular}


Estas frecuencias absolutas han sido transformadas a frecuencias relativas y utilizando la razón crítica de diferencias de porcentajes, hemos contrastado la diferencia de respuestas en función al grado de instrucción, tal como puede verse en las tablas 2, 3 y 4.

TABLA 2: Resultados de la pregunta 1 ¿Ser Ciudadano Es?

\begin{tabular}{|c|c|c|c|c|c|}
\hline & \\
\hline & $\begin{array}{c}\text { Est. } \\
\text { Secundaria }\end{array}$ & $\begin{array}{l}\text { Est. } \\
\text { versitarios }\end{array}$ & dif & $\mathrm{EE}$ & $\mathrm{Rc}$ \\
\hline R1 Tener el DNI & $100 \%$ & $8 \%$ & 92 & 4.52 & $20.35^{*}$ \\
\hline R2 Tener derechos & $0 \%$ & $70 \%$ & 70 & 7.64 & $9.17 *$ \\
\hline R3 Tener derechos y obligaciones & $0 \%$ & $22 \%$ & 22 & 6.90 & $3.19^{*}$ \\
\hline
\end{tabular}

De la tabla se desprende que la representación ciudadana es relativamente alta hacia el contenido concreto en estudiantes de secundaria, puesto que para ellos ser ciudadano es tener DNI, lo que ha disminuido en estudiantes universitarios, pero se conserva un $8 \%$ preocupante. Los estudiantes de secundaria no responden que ser ciudadano significa tener derechos y luego, además tener obligaciones, hecho que en los universitarios si se presenta de manera significativa, aun cuando el $70 \%$ dice que es tener derechos, en tanto que sólo un $22 \%$ afirma que ser ciudadano es tener derechos y obligaciones, dato que en alguna medida puede estar asociado al desapego con las instituciones con las responsabilidades que incluyen amigos, compañeros de trabajo hasta la pareja, que es de interés continuar investigándolo.

Tabla 3: Resultados de la pregunta 2 ¿Te sientes ciudadano?

\begin{tabular}{|c|c|c|c|c|c|}
\hline & $\begin{array}{c}\text { Est. } \\
\text { Secundaria }\end{array}$ & $\begin{array}{l}\text { Est. } \\
\text { iversitarios }\end{array}$ & dif & $\mathrm{EE}$ & $\mathrm{Rc}$ \\
\hline$\overline{\mathrm{R} 1 \mathrm{Si}}$ & $100 \%$ & $56 \%$ & 44 & 8.27 & $5.32 *$ \\
\hline R2 En parte & $0 \%$ & $44 \%$ & 44 & 8.27 & $5.32 *$ \\
\hline R3 No & $0 \%$ & $0 \%$ & 0 & 0 & 0 \\
\hline
\end{tabular}

De la tabla se desprende que el $100 \%$ de secundarios se sienten por sólo el $56 \%$ de donde el $44 \%$ de estos se sienten sólo en parte ciudadanos, estos porcentajes son significativos, dato que indica la carencia de sentimientos de pertenencia con su comunidad.

Tabla 4: Resultados de la pregunta 3 ¿Qué esperas de ser ciudadano en el Perú?

\begin{tabular}{lccccc}
\cline { 2 - 5 } & $\begin{array}{c}\text { Est. } \\
\text { Secundaria }\end{array}$ & $\begin{array}{c}\text { Est. } \\
\text { Rniversitarios }\end{array}$ & dif & EE & Rc \\
\hline R1 Tener comodidades & $87 \%$ & $50 \%$ & 37 & 9.97 & $3.71 *$ \\
R2 Resolver problemas del país & $13 \%$ & $50 \%$ & 37 & 9.97 & $3.71 *$ \\
\hline *) $\boldsymbol{p}<\mathbf{0 . 0 5}$ & & & & &
\end{tabular}

De los datos se desprende que los estudiantes de secundaria son significativamente apegados a los bienes materiales y placenteros por el hecho de ser ciudadano en el Perú, 
los estudiantes universitarios no es tan menor este porcentaje, dividiendo al grupo en dos mitades equivalentes; así mismo los universitarios en un 50\% asumen que deben resolver problemas del país, porcentaje polarizado en este segmento poblacional. En tanto que en los estudiantes de secundaria sólo se encuentra en el $13 \%$, el $87 \%$ de los estudiantes de secundaria no la asumen como una posibilidad. Por este dato encontramos que la posibilidad de un país grande a partir del sentimiento ciudadano de convertirse en solucionador de problemas es poco probable.

\section{Confianza en la democracia}

En cuanto a la confianza en la democracia se aprecia que no hay diferencia significativa entre ambos grupos de estudiantes; donde los estudiantes universitarios y los estudiantes de secundaria presentan medias similares en la confianza hacia las instituciones Instituciones del aparato estatal y civil; donde los valores promedios corresponden en cada uno de los grupos al grado cuatro de una escala de 10, ubicando a ambos grupos-con tendencia hacia la desconfianza, tal como puede verse en la tabla 5.

TABLA 5: Confianza en la democracia en estudiantes de secundaria y universitario de Lima Metropolitana

\begin{tabular}{cccc} 
& Secundarios & Universitarios \\
\hline Media & 44.1 & & 43.6 \\
Varianza & 98.2 & & 63.6 \\
$\mathrm{~N}$ & 36 & 0.47 & 36 \\
\hline Dif & & 2.12 & \\
EE & & 0.22 & \\
tc & 2.03 & \\
t & & 70 & \\
gl & & 0.05 & \\
N. Confianza & & Se acepta Ho
\end{tabular}




\section{DISCUSIÓN}

Partimos de que la presente investigación intentó explorar la existencia de la relación entre la representación de ciudadanía y confianza en la democracia. En primer lugar era posible aproximarse al conocimiento de la descripción de la representación ciudadana, habiendo partido de la idea de que la población peruana presenta diferentes niveles de ciudadanía, tal como lo expone Sinesio López ${ }^{5}$. En segundo lugar debíamos medir la confianza en la democracia, lo cual fue hecho a través de recoger las calificaciones asignadas a los diferentes componentes de la democracia. Los resultados en cuanto a la representación de ciudadanía nos entregan diferencias entre ambos grupos, al punto de que los alumnos de secundaria no cuestión la posesión de ciudadanía, y esta es identificada con tener el DNI. Aunque ninguno de ellos todavía lo tenía, se visualiza como ciudadano total. En cuanto a la tenencia del DNI, este es un indicador de tipo concreto, al ser palpable y estar relacionado con el pasar de una edad, y bastar este requisito, además de tener una partida de nacimiento debidamente registrada. Se debía esperar este carácter más bien concreto. En nuestra opinión si, toda vez que hay la aspiración de demostrar que se es ciudadano, con el conocimiento de que abre algunas puertas, cerradas a los menores de edad, lo cual estaría relacionado con la demanda de libertad y de ser reconocido como adulto. Los estudiantes universitarios en su mayoría ya han pasado esta etapa o gozan de una capacidad de movimiento distinta al estudiante de secundaria.

En torno a la identificación de la ciudadanía con los deberes y derechos. Presupone un nivel de abstracción mayor, relacionado con el ejercicio de la autonomía y la exigencia de tomar más decisiones. Se requiere de evaluar las consecuencias de los actos y esto es más próximo al universitario que al escolar.

Sobre la confianza en la democracia, medida por la calificación asignada a las instituciones y componentes general de la democracia, se puede observar que la pérdida de aprobación de estas por la población en diferentes segmentos, tanto etáreos como educacionales se ha homogenizado, encontrándose que de 10 puntos posibles el promedio es igual a lo cual indica que la recuperación de la confianza será un trabajo largo que implicará a más de una generación. La medición fue hecha en un momento en el que la imagen que se proyectaba del país era el de instituciones que no funcionaban, que estaban dominadas por la corrupción, que habían sido utilizadas para intereses particulares, y que aunque se hubiese recuperado la institucionalidad, aún había que _ pasar por un largo proceso de transición. Con una población que esperaba lograr una mayor capacidad adquisitiva y pruebas tangibles de que esto estaba ocurriendo.

La cobertura realizada por los medios masivos han contribuido a crear una opinión que ya es compartida por escolares y universitarios, aun cuando analicen su ciudadanía desde perspectivas diferentes. En cuanto a la relación entre ambos factores se encuentra que esta ausente, porque no hay diferencias en cuanto a la confianza en la democracia. ¿Era esperable esto? Realmente no se esperaba este resultado. Analizando el panorama que presenta esta situación, es factible sostener que el proceso de llegar a ser ciudadano y por ende formar una representación, es impulsado por el grupo social pero decidido en gran parte por el individuo, mientras que la confianza en la democracia puede empezar en el individuo pero tiene la tendencia a ser determinado socialmente. 


\section{CONCLUSIONES}

- El nivel de confianza en la democracia de los jóvenes escolares y de estudiantes universitarios es bajo, expresado centralmente en identificar al gobierno actual con el sistema democrático.

- La representación de ciudadanía de los jóvenes escolares es diferente a los estudiantes universitarios, esto posiblemente debido al pronto ingres-o al campo laboral y al compromiso personal que tienen.

- El nivel educativo no tiene efecto sobre la confianza en la democracia.

- Los alumnos de los últimos años de secundaria no problematizan el ejercicio de sus derechos y muy pocos expresan nociones relacionadas con la confianza en la democracia.

- La representación de ciudadanía que se ha formado cada individuo está referida a grados, tales como ciudadanía completa o incompleta o no ciudadano, ésta última más bien es infrecuente

\section{Sugerencias}

- La situación de nuestro país requiere como un elemento importante la confianza en su sistema democrático, hecho que es una condición para hacerlo viable y sostenible en el futuro

- La Psicología Política y la puesta a prueba de sus diversas teorías y métodos es un reto para la disciplina en la que nos desenvolvemos

\section{REFERENCIAS}

1. López S. El Mapa de la Ciudadanía en el Perú (1997)

2. Moscovici, S. Psicología Social. Barcelona: Ed. Piados (1961)

3. Ponce C, Aliaga]. (Revista de Psicología (1999) III(5): 113

4. Murakami, Y. La democracia según $C$ y D: Un estudio de la conciencia y el comportamiento político de los sectores populares de Lima. Lima: Ed. Instituto de Estudios Peruanos (2000)

5. López, S. Las ciudadanías imaginadas. La ciudadanía, las diferencias, la modernización y la democratización. En: Ciudadanos reales e imaginarios. Rev. Cuestión de Estado (1997) 20: 79-119, 385- 402

6. Franco, C. La otra modernidad Lima: Ed. CEDES (1997)

7. Bobbio, N. «Democracia representativa y democracia directa». En: Bobbio, N.: El futuro de la democracia. México: Fondo de Cultura Económica.(1984) 